\title{
Essential Self-Adjointness of Operators in Ordered Hilbert Space
}

\author{
William G. Faris \\ Battelle, Advanced Studies Center, Geneva, Switzerland
}

Received October 15, 1972

\begin{abstract}
Let $H_{0} \geqq 0$ be a self-adjoint operator acting in a space $L^{2}(M, \mu)$. It is assumed that $H_{0} e=0$, where $e$ is strictly positive, and that $\exp \left(-t H_{0}\right)$ is positivity preserving for $t \geqq 0$. Let $V$ be a real function on $M$ such that its positive part is in $L^{2}\left(M, e^{2} \mu\right)$ and its negative part is relatively small with respect to $H_{0}$. Then $H=H_{0}+V$ is essentially selfadjoint on the intersection of the domains of $H_{0}$ and $V$. This result is applied to Schrödinger operators and to quantum field Hamiltonians.
\end{abstract}

\section{Introduction}

Let $H_{0} \geqq 0$ and $V$ be self-adjoint operators. If $V$ is sufficiently regular and if the negative part of $V$ is suitably small, then the (quadratic form) sum $H=H_{0}+V$ is a uniquely defined self-adjoint operator [6; Chapter VI]. There need be no restriction on the size of the positive part of $V$. However it does not follow that there are very many vectors in the intersection of the domains of $H_{0}$ and $V$. Additional conditions are needed to ensure that $H$ be essentially self-adjoint on the intersection of the domains, and that is the subject of this paper.

The main results are the essential self-adjointness theorem for operators acting in an ordered Hilbert space (Theorem 4.4) and its application to Schrödinger operators (Theorem 5.2). This application gives a particularly simple proof of essential self-adjointness for Schrödinger operators without use of partial differential equation methods. The proof of the theorem is based on a theory of contractive semigroups and an extension of a lemma of Davies [1].

A theory of essential self-adjointness using $L^{p}$ space methods and hypercontractive semigroups was developed for use in quantum field theory $[10,12,13,15,5]$ and was applied to Schrödinger operators by Simon [13]. Simon treats only potentials $V$ which are bounded below. They are required to be locally in $L^{2}$ and to satisfy a growth condition at infinity. By using partial differential equation techniques Davies [1] was able to deal with $V$ which are unbounded below. The positive part of $V$ is required to be locally in $L^{p}$ for some $p>\frac{n}{2}, p \geqq 2$ (where $n$ is the 
dimension of the space). Thus it need not satisfy a growth condition at infinity, but the restriction on local singularities is stronger (when $n \geqq 4$ ). In the present paper essential self-adjointness for Schrödinger operators is proved under conditions similar to Simon's, but allowing $V$ to be unbounded below.

After the work on this paper was completed, the author learned that Kato [7] has recently obtained what must be the ultimate result of this nature. The potential $V$ is allowed to be rather severely unbounded below and the positive part is required only to be locally in $L^{2}$. His proof is based on partial differential equation techniques. The present approach retains the advantage of simplicity and generality; it is not restricted to Schrödinger operators. In fact the abstract theorem applies just as well to some of the quantum field Hamiltonians studied by Glimm and Jaffe (Theorem 6.1).

\section{Form Sums}

In this section we set forth some basic facts about addition of selfadjoint operators.

If $A$ is a self-adjoint operator acting in a Hilbert space $\mathscr{H}$, then its domain $\mathscr{D}(A) \subset \mathscr{H}$ is also a Hilbert space with the graph norm $\|f\|_{\mathscr{D}}^{2}$ $=\|A f\|^{2}+\|f\|^{2}$. Let $\mathscr{E}$ be a linear subspace of $\mathscr{D}(A)$. Then $A$ is said to be essentially self-adjoint on $\mathscr{E}$ if $\mathscr{E}$ is dense in $\mathscr{D}(A)$.

Let $H_{0}$ and $V$ be self-adjoint operators. Assume that there is a selfadjoint operator $H$ with $\mathscr{D}\left(H_{0}\right) \cap \mathscr{D}(V) \subset \mathscr{D}(H)$ such that $H$ agrees with $H_{0}+V$ on $\mathscr{D}\left(H_{0}\right) \cap \mathscr{D}(V)$. It is a consequence of a theorem of Trotter $[9 ; \S 8]$ that if $H$ is essentially self-adjoint on $\mathscr{D}\left(H_{0}\right) \cap \mathscr{D}(V)$, then $\exp (i t H) f=\lim _{n \rightarrow \infty}\left(\exp \left(i \frac{t}{n} H_{0}\right) \exp \left(i \frac{t}{n} V\right)\right)^{n} f$ for all $f \in \mathscr{H}$. Thus essential self-adjointness is a sufficient condition for the validity of this perturbation formula. The interesting point is that this condition may be satisfied even when $V$ is not relatively bounded with respect to $H_{0}$. Thus the Trotter formula may hold in situations where other perturbation formulas fail. From the present point of view the importance of proving essential self-adjointness is that it implies the validity of the Trotter product formula.

Definition 2.1. Let $\mathscr{H}$ be a Hilbert space and $A$ be a self-adjoint operator acting in $\mathscr{H}$. The form domain $\mathscr{Q}=\mathscr{Q}(A) \subset \mathscr{H}$ is the domain of the operator $|A|^{\frac{1}{2}}$ acting in $\mathscr{H}$, with the norm $\|f\|_{\mathscr{Q}}=\left\|(1+|A|)^{\frac{1}{2}} f\right\|$. The form dual $2^{*}$ is the completion of $\mathscr{H}$ in the norm $\|g\|_{2^{*}}$ $=\left\|(1+|A|)^{-\frac{1}{2}} g\right\|$.

Notice that $\mathscr{D}(A) \subset \mathscr{Q}(A)$; the form domain of $A$ is larger than the domain of $A$. 
If $g \in \mathscr{H}$ and $f \in \mathscr{Q}$, then the inner product $\langle g, f\rangle$ of the Hilbert space $\mathscr{H}$ satisfies $|\langle g, f\rangle| \leqq\|g\|_{2^{*}}\|f\|_{2^{2}}$. Thus there is a natural definition (by continuity) of $\langle g, f\rangle$ for $g \in \mathscr{2}^{*}, f \in \mathcal{Q}$.

Definition 2.2. Let $A$ be a self-adjoint operator acting in $\mathscr{H}$ with form domain 2 . Denote also by $A$ its extension by continuity to an operator $A: \mathscr{2} \rightarrow 2^{*}$. The form of $A$ is the sesquilinear form on $2 \times 2$ given by $\langle A h, f\rangle, h \in \mathscr{Q}, f \in \mathscr{Q}$.

If $A$ and $B$ are self-adjoint operators, we write $B \leqq A$ if $\mathscr{2}(A) \subset \mathscr{Q}(B)$ and $\langle B f, f\rangle \leqq\langle A f, f\rangle$ for all $f \in \mathscr{Q}(A)$. In the next two propositions we record known facts about this order relation in the form in which we need them.

Proposition 2.3. Let $A$ and $B$ be self-adjoint operators with $0 \leqq B \leqq A$. Then $0 \leqq B^{r} \leqq A^{r}$ for all $r$ with $0<r<1$.

Proof. First consider the special case when there exists a $c$ with $0<c \leqq B \leqq A$. Then $(A+t)^{-1} \leqq(B+t)^{-1}$ for all $t \geqq 0$, so using $A^{-r}=\frac{1}{\pi} \sin (\pi r) \int_{0}^{\infty} t^{-r}(A+t)^{-1} d t[6$; Chapter $\mathrm{V}, \S 11]$ and the corresponding representation for $B^{-r}$ we see that $A^{-r} \leqq B^{-r}$, and so $B^{r} \leqq A^{r}$.

In the general case we have $0 \leqq B^{r} \leqq(B+\varepsilon)^{r} \leqq(A+\varepsilon)^{r}$ for every $\varepsilon>0$. Hence $B^{r} \leqq A^{r}$, by the monotone convergence theorem.

Proposition 2.4. Let $A$ and $B$ be self-adjoint operators with $0<c$ $\leqq B \leqq A$. Then $\log B \leqq \log A$.

Proof. The proof is similar to the previous proposition [3].

Definition 2.5. Let $A$ and $B$ be self-adjoint operators acting in the Hilbert space $\mathscr{H}$. Consider the corresponding forms $\langle A f, g\rangle$ and $\langle B f, g\rangle$. If there exists a self-adjoint operator $C$ such that $\mathscr{Q}(C)=\mathscr{Q}(A)$ $\cap \mathscr{Q}(B)$ and such that its form satisfies $\langle C f, g\rangle=\langle A f, g\rangle+\langle B f, g\rangle$, then this operator $C$ will be called the form sum of $A$ and $B$.

Proposition 2.6. Let $C$ be the form sum of $A$ and $B$. Then $\mathscr{D}(A) \cap \mathscr{D}(B)$ $C \mathscr{D}(C)$.

Proof. Let $f$ be in $\mathscr{D}(A) \cap \mathscr{D}(B)$. Then $A f \in \mathscr{H}$ and $B f \in \mathscr{H}$, so $g \mapsto\langle C f, g\rangle=\langle A f, g\rangle+\langle B f, g\rangle$ is a functional on $\mathscr{2}(C)$ which extends by continuity to $\mathscr{H}$. This implies that $C f \in \mathscr{H}$, so $f \in \mathscr{D}(C)$.

If $C$ is the form sum of $A$ and $B$ we write $C=A+B$, even though in general it is possible that $\mathscr{D}(A) \cap \mathscr{D}(B)=\{0\}$.

Theorem 2.7. [6; Chapter V]. Let $\mathscr{H}$ be a Hilbert space and $H_{0} \geqq 0$ be a self-adjoint operator acting in $\mathscr{H}$. Let $U$ and $W$ be self-adjoint operators acting in $\mathscr{H}$. Assume that $U \geqq 0$ and that $\mathscr{2}\left(H_{0}\right) \cap \mathscr{Q}(U)$ is dense in $\mathscr{H}$. Assume that there exists $a<1$ and $b$ such that $\pm W \leqq a\left(H_{0}+b\right)$ (in particular $\mathscr{2}(W) \supset \mathscr{Q}\left(H_{0}\right)$ ). Then there exists a self-adjoint operator $H$ 
which is the form sum $H=\left(H_{0}+U\right)+W$. Also $\mathscr{2}(H)=\mathscr{Q}\left(H_{0}\right) \cap \mathscr{Q}(U)$ and $H$ is bounded below, in fact, $H \geqq-b$.

Proof. The dense subspace $2\left(H_{0}\right) \cap \mathscr{Q}(U)$ with the inner product $\left\langle\left(H_{0}+U+1\right) f, g\right\rangle$ is a Hilbert space. Thus $H_{0}+U$ is self-adjoint with $\mathscr{2}\left(H_{0}+U\right)=\mathscr{Q}\left(H_{0}\right) \cap \mathscr{Q}(U)[9 ; \S 7$, Theorem 2].

Let $H_{1}=H_{0}+U$ and $2=2\left(H_{1}\right)$. Then $\pm W=a\left(H_{0}+b\right) \leqq a\left(H_{1}+b\right)$, that is, $W: \mathscr{Q} \rightarrow \mathscr{2}^{*}$ with $\left\|\left(H_{1}+b\right)^{-\frac{1}{2}} W\left(H_{1}+b\right)^{-\frac{1}{2}}\right\| \leqq a<1$. Hence $\left(H_{1}+W+b\right)^{-1}=\left(H_{1}+b\right)^{-\frac{1}{2}}\left[1+\left(H_{1}+b\right)^{-\frac{1}{2}} W\left(H_{1}+b\right)^{-\frac{1}{2}}\right]^{-1}\left(H_{1}+b\right)^{-\frac{1}{2}}$ exists as an operator from $2^{*}$ to 2 . In particular, it sends $\mathscr{H}$ into $\mathscr{D}\left(H_{1}+W\right) \subset \mathscr{Q}$ and so $H_{1}+W$ is self-adjoint with $\mathscr{2}\left(H_{1}+W\right)=\mathscr{2}\left(H_{1}\right)$.

\section{Ordered Hilbert Space}

In this section we present some elementary aspects of the theory of ordered Hilbert spaces [11; Chapter V]. Since it is convenient to deal with order in real spaces, we begin with a brief discussion of the reduction of the problem from complex spaces to real spaces.

Definition 3.1. Let $\mathscr{W}$ be a complex Hilbert space. Then $J: \mathscr{W} \rightarrow \mathscr{W}$ is a conjugation if $J$ is anti-linear $\left(J(a f+b g)=a^{*} J(f)+b^{*} J(g)\right)$, antiunitary $\left(\langle J f, J g\rangle=\langle f, g\rangle^{*}\right)$ and $J^{2}=1$. If $f \in \mathscr{W}$ and $J f=f$, then $f$ is called real (with respect to $J$ ). If $A$ is an operator acting in $\mathscr{W}$ such that $f \in \mathscr{D}(A)$ implies that $J f \in \mathscr{D}(A)$ and $J A f=A J f$, then $A$ is called real.

The set of real elements in a complex Hilbert space forms a real Hilbert space. If $A$ is real, then $A$ leaves this real Hilbert space invariant. Questions about real operators in a complex Hilbert space may often be reduced to questions about operators in a real Hilbert space.

Note in particular that if $A$ is a real self-adjoint operator with respect to the conjugation $J: \mathscr{W} \rightarrow \mathscr{W}$ then $J: \mathscr{D}(A) \rightarrow \mathscr{D}(A)$ is also a conjugation with respect to the graph inner product on $\mathscr{D}(A)$. Thus for questions of essential self-adjointness for real operators it is enough to consider only real Hilbert space.

Definition 3.2. Let $\mathscr{H}$ be a real vector space. A proper cone is a subset $\mathscr{K}$ of $\mathscr{H}$ such that $\mathscr{K}+\mathscr{K} \subset \mathscr{K}, a \mathscr{K} \subset \mathscr{K}$ for all $a \geqq 0$, and $\mathscr{K} \cap-\mathscr{K}=\{0\}$. If a proper cone $\mathscr{K} \subset \mathscr{H}$ is given, then $\mathscr{H}$ is called an ordered vector space and $\mathscr{K}$ is called the positive cone of $\mathscr{H}$. If $f$ and $g$ are elements of $\mathscr{H}$, we write $f \leqq g$ whenever $g-f$ belongs to the positive cone $\mathscr{K}$. If $\mathscr{H}$ is a real Banach space and the positive cone $\mathscr{K}$ is closed in $\mathscr{H}$, then $\mathscr{H}$ is called an ordered Banach space.

Definition 3.3. Let $\mathscr{H}$ be an ordered Banach space and let $e$ be a positive element of $\mathscr{H}$. If $f \in \mathscr{H}$, set $\|f\|_{e}=\inf \{t \geqq 0: \pm f \leqq t e\}$. Then $\mathscr{L}(e)$ is defined to be the space of all $f$ in $\mathscr{H}$ such that $\|f\|_{e}<\infty$. 
Proposition 3.4. [11; Chapter V]. The space $\mathscr{L}(e)$ is a normed space and the ball $\|f\|_{e} \leqq c$ is a closed subset of $\mathscr{H}$ for every $c \geqq 0$.

Proof. Since the positive cone is closed in $\mathscr{H}$, the infimum in the definition of $\|f\|_{e}$ is assumed. Thus $\|f\|_{e} \leqq c$ if and only if $\pm f \leqq c e$. From this it follows that $\|f\|_{e}$ is a norm: if $\|f\|_{e}=0$, then $\pm f \leqq 0$, so $f=0$. The fact that the ball $\|f\|_{e} \leqq c$ is closed also follows from the fact that the positive cone is closed in $\mathscr{H}$.

Definition 3.5. Let $\mathscr{H}$ be an ordered vector space and let $A$ be a linear operator acting in $\mathscr{H}$. Then $A$ is said to be positivity preserving if $f \geqq 0$ implies $A f \geqq 0$.

Proposition 3.6. Let $\mathscr{H}$ be an ordered Banach space. Let $A: \mathscr{H} \rightarrow \mathscr{H}$ be a positivity preserving operator. Assume Ae $\leqq$. Then $A$ leaves $\mathscr{L}(e)$ invariant and $\|A f\|_{e} \leqq\|f\|_{e}$.

Proof. Since $\pm f \leqq\|f\|_{e} e$, we have $\pm A f \leqq\|f\|_{e} A e \leqq\|f\|_{e} e$.

Definition 3.7. Let $\mathscr{H}$ be an ordered vector space. An absolute value is a function $f \mapsto|f|$ from $\mathscr{H}$ to $\mathscr{H}$ such that $\pm f \leqq|f|$.

It follows from $-|f| \leqq f \leqq|f|$ that $|f| \geqq 0$ and that $|f|=0$ implies $f=0$. (Notice that we do not assume that $\pm f \leqq g$ implies $|f| \leqq g$.) We define $f_{ \pm}=\frac{1}{2}(|f| \pm f)$. Then $f_{ \pm} \geqq 0$ and $f=f_{+}-f_{-},|f|=f_{+}+f_{-}$.

Definition 3.8. Let $\mathscr{H}$ be a real Hilbert space which is an ordered vector space. Assume that the positive cone is closed in $\mathscr{H}$. Then $\mathscr{H}$ is called an ordered Hilbert space if $f \geqq 0$ and $g \geqq 0$ implies $\langle f, g\rangle \geqq 0$ and there is an absolute value defined on $\mathscr{H}$ such that $\||f|\|=\|f\|$.

It is a consequence of the first part of the definition that $\pm f \leqq g$ implies $\|f\| \leqq\|g\|$. In fact $\|g\|^{2}-\|f\|^{2}=\langle g+f, g-f\rangle \geqq 0$. The condition $\||f|\|=\|f\|$ of the second part of the definition is equivalent to $\left\langle f_{+}, f_{-}\right\rangle=0$.

The obvious example of an ordered Hilbert space is $\mathscr{H}=L^{2}(M, \mu)$, where $\mu$ is a measure in the measure space $M$. (The space consists of real functions and $f \leqq g$ means $f(p) \leqq g(p)$ for almost every $p$.) Another example is the space of self-adjoint Hilbert-Schmidt operators. This space is not a lattice and its order structure has other unfamiliar properties. For instance, if $u=\left(\begin{array}{rr}1 & 0 \\ 0 & -1\end{array}\right)$ and $v=\left(\begin{array}{ll}3 & 2 \\ 2 & 2\end{array}\right)$, then $\pm u \leqq v$ but it is false
that $|u| \leqq v$.

Proposition 3.9. [1]. Let $\mathscr{H}$ be an ordered Hilbert space and $A: \mathscr{H} \rightarrow \mathscr{H}$ and $B: \mathscr{H} \rightarrow \mathscr{H}$ be positivity preserving. Assume that $f \geqq 0$ implies $B f \leqq A f$. Then $\|B\| \leqq\|A\|$.

Proof. Since $\pm B f \leqq B|f| \leqq A|f|$, we see that $\|B f\| \leqq\|A|f|\|$ $\leqq\|A\|\||f|\|=\|A\|\|f\|$. 
Proposition 3.10. Let $\mathscr{H}$ be an ordered Hilbert space. Let $A$ be a self-adjoint positivity preserving operator acting in $\mathscr{H}$. Assume that $h \in \mathscr{D}(A)$ implies $|h| \in \mathscr{D}(A)$. If $g \in \mathscr{D}(A)$ and $\pm f \leqq g$, then $f \in \mathscr{D}(A)$.

Proof. If $g \in \mathscr{D}(A)$ we have $\pm\langle f, A h\rangle= \pm\left\langle f, A h_{+}\right\rangle \mp\left\langle f, A h_{-}\right\rangle$ $\leqq\left\langle g, A h_{+}\right\rangle+\left\langle g, A h_{-}\right\rangle=\langle g, A|h|\rangle$. Thus $|\langle f, A h\rangle| \leqq\langle A g,|h|\rangle \leqq\|A g\|\|h\|$. It follows that $h \mapsto\langle f, A h\rangle$ is continuous, so $f \in \mathscr{D}(A)$.

Corollary 3.11. Let $e \in \mathscr{H}$ with $e \geqq 0$. Let $U$ be a self-adjoint positivity preserving operator acting in $\mathscr{H}$ such that $h \in \mathscr{D}(U)$ implies $|h| \in \mathscr{D}(U)$ and such that $e \in \mathscr{D}(U)$. Then $\mathscr{L}(e) \subset \mathscr{D}(U)$.

\section{Essential Self-Adjointness}

In this section we prove a theorem on existence of form sums and a theorem on essential self-adjointness of operator sums. The theorem on form sums (Theorem 4.1) is a direct consequence of a more general result (Theorem 2.7). It is given here only to show the parallel with the theorem on operator sums (Theorem 4.4). The theorem on operator sums is proved by means of a theory of contractive semigroups (Lemma 1) and an extension of a method of Davies for obtaining quadratic estimates (Lemma 2).

Theorem 4.1. Let $\mathscr{H}$ be an ordered Hilbert space. Let $e \geqq 0$ be an element of $\mathscr{H}$ such that $\mathscr{L}(e)$ is dense in $\mathscr{H}$. Let $H_{0} \geqq 0$ be a self-adjoint operator acting in $\mathscr{H}$ such that $H_{0} e=0$. Assume that $\exp \left(-t H_{0}\right)$ is positivity preserving for $t \geqq 0$. Let $U$ and $W$ be commuting self-adjoint operators acting in $\mathscr{H}$ and set $V=U+W$. Assume that $U \geqq 0$ and $\mathscr{L}(e) \subset \mathscr{Q}(U)$. Assume also that there exists $a<1$ and $b$ such that $\pm W \leqq a\left(H_{0}+b\right)$. Then the form sum $H=H_{0}+V$ is self-adjoint and bounded below.

Proof. In order to apply Theorem 2.7 we need only show that $\mathscr{2}\left(H_{0}\right) \cap \mathscr{Q}(U)$ is dense in $\mathscr{H}$. Let $\mathscr{E}=\exp \left(-H_{0}\right) \mathscr{L}(e)$. Then $\mathscr{E} \subset \mathscr{D}\left(H_{0}\right) \subset \mathscr{Q}\left(H_{0}\right)$. Since $\exp \left(-t H_{0}\right)$ is positivity preserving and $\exp \left(-t H_{0}\right) e=e, \exp \left(-t H_{0}\right)$ sends $\mathscr{L}(e)$ into $\mathscr{L}(e)$ by Proposition 3.6. Hence $\mathscr{E} \subset \mathscr{L}(e) \subset \mathscr{D}(U)$. To see that $\mathscr{E}$ is dense in $\mathscr{H}$, we observe that if $h \perp \mathscr{E}$, then $\exp \left(-H_{0}\right) h \perp \mathscr{L}(e)$, so $\exp \left(-H_{0}\right) h=0, h=0$.

Since $U$ and $W$ commute, $\mathscr{Q}(U) \cap \mathscr{2}(W) \subset \mathscr{Q}(V)$ and $\mathscr{2}(V) \cap \mathscr{Q}(W) \subset \mathscr{Q}(U)$. Hence $2(H)=\mathscr{Q}\left(H_{0}\right) \cap \mathscr{Q}(V)$ and $H=H_{0}+V$. This completes the proof of the theorem.

In the following corollary we let $U, W, V$ denote real measurable functions on $M$ and also the corresponding multiplication operators on $L^{2}(M, \mu)$. 
Corollary 4.2. Let $\mu$ be a measure in $M$ and let $\mathscr{H}=L^{2}(M, \mu)$. Let $e$ be an element of $\mathscr{H}$ such that $e(p)>0$ for almost every $p$ in $M$. Let $H_{0}$ be a self-adjoint operator acting in $\mathscr{H}$ such that $\mathrm{H}_{0} e=0$. Assume that $\exp \left(-t H_{0}\right)$ is positivity preserving for $t \geqq 0$. Let $U$ and $W$ be real measurable functions on $M$ and set $V=U+W$. Assume that $U \geqq 0$ and that $U \in L^{1}\left(M, e^{2} \mu\right)$. Assume also that there exist $a<1$ and $\bar{b}$ such that $\pm W \leqq a\left(H_{0}+b\right)$. Then the form sum $H=H_{0}+V$ is self-adjoint and bounded below.

Proof. Since $\exp \left(-t H_{0}\right)$ is positivity preserving, it is a real operator (with respect to complex conjugation). Hence $H_{0}$ is real. Thus we may consider $H_{0}$ and $V$ as operators in a real Hilbert space.

We need only show that $\mathscr{L}(e)$ is dense in $\mathscr{H}$. But if $h \perp \mathscr{L}(e)$, then $h \perp e \operatorname{sign}(h)$, so $|h| \perp e$. Since $e>0$ almost everywhere, this implies that $h=0$.

Definition 4.3. Let $U \geqq 0$ be a self-adjoint operator. The truncated operator $U_{k}$ is defined by $U_{k}=U$ on the subspace where $U_{k} \leqq k, U_{k}=0$ on the orthogonal complement.

Theorem 4.4. Let $\mathscr{H}$ be an ordered Hilbert space. Let $e \geqq 0$ be an element of $\mathscr{H}$ such that $\mathscr{L}(e)$ is dense in $\mathscr{H}$. Let $H_{0}$ be a self-adjoint operator acting in $\mathscr{H}$ such that $H_{0} e=0$. Assume that $\exp \left(-t H_{0}\right)$ is positivity preserving for $t \geqq 0$. Let $U$ and $W$ be commuting self-adjoint operators acting in $\mathscr{H}$ and set $V=U+W$. Assume that $U \geqq 0$, that $\mathscr{L}(e) \subset \mathscr{D}(U)$, and that for each $k \exp \left(-t U_{k}\right)$ and $1-\exp \left(-t U_{k}\right)$ are positivity preserving for $t \geqq 0$. Assume also that $|W|$ is positivity preserving, that its domain is invariant under the absolute value, and that there exist constants $a<1$ and $b$ such that $W^{2} \leqq a^{2}\left(H_{0}+b\right)^{2}$. Let $H=H_{0}+V$. Then $H$ is essentially self-adjoint on $\mathscr{D}\left(H_{0}\right) \cap \mathscr{D}(V)$.

Remark. In the statement of the theorem we have assumed that $W$ satisfies the second order estimate $W^{2} \leqq a^{2}\left(H_{0}+b\right)^{2}$ and consequently $\mathscr{D}(W) \supset \mathscr{D}\left(H_{0}\right)$. It follows from Proposition 2.3 that $W$ satisfies the first order estimate $|W| \leqq a\left(H_{0}+b\right)$ and so $\mathscr{Z}(W) \supset \mathscr{Z}\left(H_{0}\right)$. Thus $H$ is selfadjoint and bounded below, by Theorem 4.1 .

Lemma 1. Let $H_{1}=H_{0}+U$. Then $H_{1}$ is essentially self-adjoint on $\mathscr{D}\left(H_{1}\right) \cap \mathscr{D}(U)$.

Proof. Let $U_{k}$ be the truncated operator: $U_{k}=U$ where $U \leqq k$, 0 otherwise. Set $H_{1 k}=H_{0}+U_{k}$. Then $H_{1 k}$ is self-adjoint with $\mathscr{D}\left(H_{1 k}\right)$ $=\mathscr{D}\left(H_{0}\right)$, since $U_{k}$ is bounded. It follows from the Trotter product formula $[9 ; \S 8]$ that $\exp \left(-t H_{1 k}\right) f=\lim _{n \rightarrow \infty}\left(\exp \left(-\frac{t}{n} H_{0}\right) \exp \left(-\frac{t}{n} U_{k}\right)\right)^{n} f$
for all $f \in L^{2}$ and $t \geqq 0$. 
We also have $\exp \left(-t H_{1}\right) f=\lim _{n \rightarrow \infty} \exp \left(-t H_{1 k}\right) f$. In fact $U_{k} \uparrow U$, so $H_{1 k} \uparrow H_{1}$ in the sense of quadratic forms. This implies [6; Theorem VIII-3.13] [3] strong resolvent convergence. It follows [6; Theorem IX-2.16] that the corresponding semigroups converge strongly.

Since $\exp \left(-t H_{0}\right)$ is positivity preserving and $\exp \left(-t H_{0}\right) e=e$, it follows by Proposition 3.6 that $\exp \left(-t H_{0}\right)$ is a contraction on $\mathscr{L}(e)$. Also $\exp \left(-t U_{k}\right)$ is positivity preserving and $\exp \left(-t U_{k}\right) e \leqq e$ (since $1-\exp \left(-t U_{k}\right)$ is positivity preserving), so again by Proposition 3.6 $\exp \left(-t U_{k}\right)$ is a contraction on $\mathscr{L}(e)$. The fact that these semigroups are contractions on an auxiliary space justifies the term contractive semigroup [15]. It is this feature which now permits us to estimate $\exp \left(-t H_{1}\right)$ by use of the Trotter product formula.

Recall that according to Proposition 3.4 the ball $\|f\|_{e} \leqq c$ is closed in $\mathscr{H}$. Thus if $f \in \mathscr{L}(e)$ with $\|f\|_{e} \leqq c$, then $\left\|\exp \left(-t H_{1 k}\right) f\right\|_{e} \leqq c$ and so $\left\|\exp \left(-t H_{1}\right) f\right\|_{e} \leqq c$. Thus $\exp \left(-t H_{1}\right) \mathscr{L}(e) \subset \mathscr{L}(e)$ for $t \geqq 0$.

Now let $\mathscr{E}=\exp \left(-H_{1}\right) \mathscr{L}(e)$. We will see that $\mathscr{E} \subset \mathscr{D}\left(H_{0}\right) \cap \mathscr{D}(U)$ $C \mathscr{D}\left(H_{1}\right)$ and that $\mathscr{E}$ is dense in $\mathscr{D}\left(H_{1}\right)$. In order to see that $\mathscr{E}$ is dense in $\mathscr{D}\left(H_{1}\right)$ we observe that if $h \perp \mathscr{E}$ in the graph inner product on $\mathscr{D}\left(H_{1}\right)$, then $\left(1+H_{1}^{2}\right) \exp \left(-H_{1}\right) h \perp \mathscr{E}$, so $\left(1+H_{1}^{2}\right) \exp \left(-H_{1}\right) h=0, h=0$.

In order to show that $\mathscr{E} C \mathscr{D}\left(H_{0}\right) \cap \mathscr{D}(U)$, we notice that $\mathscr{E}=\exp \left(-H_{1}\right)$ - $\mathscr{L}(e) \subset \mathscr{L}(e)$ and $\mathscr{L}(e) \subset \mathscr{D}(U)$ by assumption. Thus $\mathscr{E} \subset \mathscr{D}(U)$. But also $\mathscr{E} C \mathscr{D}\left(H_{1}\right)$. So if $f \in \mathscr{E}$, then $H_{1} f$ and $U f$ are in $\mathscr{H}$. But then $g \mapsto\left\langle H_{0} f, g\right\rangle$ $=\left\langle H_{1} f, g\right\rangle-\langle U f, g\rangle$ is continuous, so $f \in \mathscr{D}\left(H_{0}\right)$. Therefore we have also shown that $\mathscr{E} \subset \mathscr{D}\left(H_{0}\right)$.

Lemma 2. There exists constants $a<1$ and $b$ such that $W^{2} \leqq a^{2}\left(H_{1}+b\right)^{2}$.

Proof. We have assumed that if $h \geqq 0$ then $0 \leqq \exp \left(-t U_{k}\right) h \leqq h$ and that if $0 \leqq f \leqq g$ then $0 \leqq \exp \left(-t H_{0}\right) f \leqq \exp \left(-t H_{0}\right) g$. It follows that $f \geqq 0$ implies $0 \leqq\left(\exp \left(-\frac{t}{n} H_{0}\right) \exp \left(-\frac{t}{n} U_{k}\right)\right)^{n} f \leqq \exp \left(-t H_{0}\right) f$. Let $n \rightarrow \infty$. We see that $0 \leqq \exp \left(-t H_{1 k}\right) f \leqq \exp \left(-t H_{0}\right) f$. Now let $k \rightarrow \infty$. We obtain $0 \leqq \exp \left(-t H_{1}\right) f \leqq \exp \left(-t H_{0}\right) f$. Finally, from the formula $\left(H_{i}+b\right)^{-1}=\int_{0}^{\infty} \exp (-b t) \exp \left(-t H_{i}\right) f d t$ we see that $0 \leqq\left(H_{1}+b\right)^{-1} f$ $\leqq\left(H_{0}+b\right)^{-1} f$.

Let $f$ be an arbitrary element of $\mathscr{H}$. Then $\pm\left(H_{1}+b\right)^{-1} f$ $\leqq\left(H_{1}+b\right)^{-1}|f| \leqq\left(H_{0}+b\right)^{-1}|f|$. Hence $\left(H_{1}+b\right)^{-1} f \in D(|W|)$, by Proposition 3.10. From Proposition 3.9 we conclude that $\left\||W|\left(H_{1}+b\right)^{-1}\right\|$ $\leqq\left\||W|\left(H_{0}+b\right)^{-1}\right\|$. This is equivalent to $\left(H_{1}+b\right)^{-1} W^{2}\left(H_{1}+b\right)^{-1}$ $\leqq\left(H_{0}+b\right)^{-1} W^{2}\left(H_{0}+b\right)^{-1}$ and the right hand side is bounded by $a^{2}$. Thus $W^{2} \leqq a^{2}\left(H_{1}+b\right)^{2}$, as was to be shown.

Notice that even though $H_{1}=H_{0}+U$ with $H_{0} \geqq 0$ and $U \geqq 0$, it is false in general that $\left(H_{0}+b\right)^{2} \leqq\left(H_{1}+b\right)^{2}$. That is why this quadratic 
estimate is more difficult than the linear estimate $\pm W \leqq a\left(H_{0}+b\right)$ $\leqq a\left(H_{1}+b\right)$ of Theorem 2.7 .

To finish the proof of the theorem, it is sufficient to recall that if $H=H_{1}+W$ and $\mathscr{D}(W) \supset \mathscr{D}\left(H_{1}\right)$ with $\left\|W\left(H_{1}+c\right)^{-1}\right\|<1$ for some $c>0$, then $\mathscr{D}(H)=\mathscr{D}\left(H_{1}\right)$ and the graph norms are equivalent. (In fact $\|(H+c) f\|=\left\|\left[1+W\left(H_{1}+c\right)^{-1}\right]\left(H_{1}+c\right) f\right\|$ and $1+W\left(H_{1}+c\right)^{-1}$ is invertible.) So if $\mathscr{E}$ is dense in $\mathscr{D}\left(H_{1}\right)$ it is dense in $\mathscr{D}(H)$. In the present case this is applied to $\mathscr{E}=\mathscr{D}\left(H_{0}\right) \cap \mathscr{D}(U)$. Since $\mathscr{D}(W) \supset \mathscr{D}\left(H_{0}\right)$ we have $\mathscr{E} \subset \mathscr{D}\left(H_{0}\right) \cap \mathscr{D}(W) \cap \mathscr{D}(U) \subset \mathscr{D}\left(H_{0}\right) \cap \mathscr{D}(V)$, so $\mathscr{D}\left(H_{0}\right) \cap \mathscr{D}(V)$ is dense in $\mathscr{D}(H)$.

Corollary 4.5. Let $\mathscr{H}=L^{2}(M, \mu)$, where $\mu$ is a measure in the measure space $M$. Let $e$ be an element of $\mathscr{H}$ such that $e(p)>0$ for almost every $p$ in $M$. Let $H_{0} \geqq 0$ be a self-adjoint operator acting in $\mathscr{H}$ such that $H_{0} e=0$. Assume that $\exp \left(-t H_{0}\right)$ is positivity preserving for $t \geqq 0$. Let $U$ and $W$ be real measurable functions on $M$ and set $V=U+W$. Assume that $U \geqq 0$ and $U \in L^{2}\left(M, e^{2} \mu\right)$. Assume also that there exist constants $a<1$ and $b$ such that the multiplication operator $W$ satisfies $W^{2} \leqq a^{2}\left(H_{0}+b\right)^{2}$. Let $H=H_{0}+V$. Then $H$ is essentially self-adjoint on $\mathscr{D}\left(H_{0}\right) \cap \mathscr{D}(V)$.

\section{Schrödinger Operators}

In this section we apply the abstract theory developed in the previous section to Schrödinger operators. The main result is Theorem 5.2. Theorem 5.1 is put in mainly to show how much easier it is to get a result which depends only on linear (rather than quadratic) estimates.

Theorem 5.1. Let $\mathscr{H}=L^{2}\left(\mathbb{R}^{n}, d x\right)$ and let $V$ be a real measurable function on $\mathbb{R}^{n}$. Assume that $V=U+W$, where $U \geqq 0$ and $U \in L^{1}$ locally on the complement of a closed set of measure zero, and where $W \in L^{p}+L^{\infty}$ for some $p \geqq \frac{n}{2}$ (and where $p \geqq 1$ if $n=1, p>1$ if $n=2$ ). Then $H=-\Delta+V$ is self-adjoint and bounded below and $2(H)=\mathscr{2}(\Delta) \cap \mathscr{2}(\mathrm{V})$.

Proof. According to Theorem 2.7 we must show that $2(\Delta) \cap \mathscr{Q}(U)$ is dense in $\mathscr{H}$ and that $\pm W \leqq a(-\Delta+b)$ for some $a<1$.

We have assumed that $U$ is locally integrable on the complement of a closed set $M$ of measure zero. Let $\mathscr{F}$ be the space of $C^{2}$ functions with compact support in the complement of $M$. Clearly $\mathscr{F} \subset \mathscr{Q}\left(H_{0}\right) \cap \mathscr{Q}(V)$ and $\mathscr{F}$ is dense in $\mathscr{H}$.

As for the estimate on $W$, it is well known [2] that under the hypotheses of the theorem for all $a>0$ there exists $b$ such that $|W| \leqq a\left(H_{0}+b\right)$. This completes the proof.

Theorem 5.2. Let $\mathscr{H}=L^{2}\left(\mathbb{R}^{n}, d x\right)$. Let $V$ be a real function on $\mathbb{R}^{n}$. Assume that $V=U+W$, where $U \geqq 0$ and $U \in L^{2}\left(\mathbb{R}^{n}, \exp (-2 a|x|) d x\right)$ 
for some $a<\infty$ and where $W \in L^{p}\left(\mathbb{R}^{n}, d x\right)+L^{\infty}\left(\mathbb{R}^{n}, d x\right)$ for some $p \geqq \frac{n}{2}$ (and $p \geqq 2$ if $n=1,2$, or $3, p>2$ if $n=4$ ). Then $H=-\Delta+V$ is essentially self-adjoint on $\mathscr{D}(\Delta) \cap \mathscr{D}(V)$.

Proof. Let $Y$ be a real function on $\mathbb{R}^{n}$ which is constant on a ball centered at the origin and is zero elsewhere. Then $-\Delta+Y$ is self-adjoint with the same domain as $-\Delta$, since $Y$ is a bounded function. By a suitable choice of $Y$ we may arrange that $-\Delta+Y$ has a strictly negative eigenvalue. We may choose it as negative as we please. Let $-c^{2}$ (where $c>0$ ) be the most negative eigenvalue of $-\Delta+Y$ and set $H_{0}=-\Delta$ $+Y+c^{2}$. Then $H_{0} \geqq 0$ and zero is an eigenvalue of $H_{0}$. It is sufficient to prove the theorem with $H_{0}$ in place of $-\Delta$.

Let $e$ be the eigenfunction of $H_{0}$ with $H_{0} e=0$. We may choose the sign of $e$ so that $e(x)>0$ for all $x$ in $\mathbb{R}^{n}$, by the Perron-Frobenius theorem or by explicit computation.

Since $\exp (t \Delta)$ is an integral operator with positive kernel for $t>0$, it is positivity preserving. Thus $\exp \left(-t H_{0}\right)$ is positivity preserving, by the Trotter product formula.

We now use our freedom to choose $c$ arbitrarily large to require that $c>a$. It may be seen from decay estimates [3] or explicit computation that this implies that $e$ satisfies an estimate $e(x) \leqq K \exp (-a|x|)$. Hence $\int U(x)^{2} e(x)^{2} d x \leqq K^{2} \int U(x)^{2} \exp (-2 a|x|) d x<\infty$, so $U \in L^{2}\left(\mathbb{R}^{n}, e(x)^{2} d x\right)$.

It is well known [2] that the hypotheses on $W$ imply that for all $a>0$ there exists $b<\infty$ such that $W^{2} \leqq a^{2}(-\Delta+b)^{2}$. However $(-\Delta+b)^{2}$ $\leqq 2\left(\left(H_{0}+b\right)^{2}+\left(Y+c^{2}\right)^{2}\right) \leqq 2\left(H_{0}+d\right)^{2}$ for $d$ sufficiently large. It follows that $W^{2} \leqq 2 a^{2}\left(H_{0}+d\right)^{2}$. The proof is completed by an application of Corollary 4.5 .

\section{Quantum Field Theory}

The Hamiltonian $H=H_{0}+V$ for a self-interacting boson field (in one space dimension with a space cutoff) is analogous to a Schrödinger operator. The Hilbert space may be represented as $L^{2}(M, \mu)$, where $M=\mathbb{R}^{\infty}$ and $\mu$ is Gaussian measure, in such a way that $H_{0} \geqq 0$ is a second order linear differential operator and $V$ is a polynomial of even degree in infinitely many variables. Such a polynomial need not be bounded below.

The foundation of the recent work on this Hamiltonian is an $L^{p}$ space estimate of Nelson [8] which may be used to prove that $H$ is self-adjoint and bounded below. Essential self-adjointness on a common domain for $H_{0}$ and $V$ was first proved (but only for $V$ of fourth degree) by Glimm and Jaffe [4] using Nelson's results and estimates on commutators. The first essential self-adjointness proof using $L^{p}$ space techniques was 
due to Rosen [10]. The $L^{p}$ space techniques were developed in an abstract setting by I. Segal $[12,13]$. The resulting theory of hypercontractive semigroups in $L^{p}$ space and its application to quantum field theory has been surveyed and extended by Simon and Hoegh-Krohn [15].

In this section we give a particularly simple proof of essential selfadjointness which uses neither estimates on commutators nor the theory of hypercontractive semigroups. It is a direct application of the same abstract theory that was applied above to Schrödinger operators.

Let $\mu$ be a measure in the measure space $M$ with $\mu(M)=1$. Let $V$ be a real measurable function on $M$. We say that $V$ satisfies the Nelson estimate [8] if there exist strictly positive constants $a, b, c, d$ such that

$$
\mu\{p: V(p) \leqq-x\} \leqq a \exp \left(-b \exp \left(c x^{d}\right)\right) .
$$

Theorem 6.1. Let $\mathscr{H}=L^{2}(M, \mu)$, where $\mu(M)=1$. Let $H_{0} \geqq 0$ be a self-adjoint operator acting in $\mathscr{H}$ with $H_{0} 1=0$. Assume that $\exp \left(-t H_{0}\right)$ is positivity preserving for all $t \geqq 0$ and that there exists $T>0$ and $r>2$ such that $\exp \left(-T H_{0}\right): L^{2} \rightarrow L^{r}$ is bounded. Assume that $V$ is a real function in $L^{2}(M, \mu)$ which satisfies the Nelson estimate. Then $H=H_{0}+V$ is self-adjoint and bounded below. In addition $H=H_{0}+V$ is essentially self-adjoint on $\mathscr{D}\left(H_{0}\right) \cap \mathscr{D}(V)$.

Proof. We apply Corollary 4.5. Write $V=V_{+}-V_{-}$, where $V_{ \pm} \geqq 0$ are the positive and negative parts of $V$. Then $V_{+} \in L^{2}(M, \mu)$. On the other hand $\exp \left(c V_{-}^{2}\right) \in L^{q}(M, \mu)$ for all $c<\infty$ and $q<\infty$, by the Nelson estimate. If we choose $q$ such that $\frac{1}{q}+\frac{1}{r}=\frac{1}{2}$, then

$$
\exp \left(c V_{-}^{2}\right) \exp \left(-T H_{0}\right): L^{2} \rightarrow L^{2}
$$

is bounded, by Hölder's inequality. Thus $\exp \left(2 c V_{-}^{2}\right) \leqq K \exp \left(2 T H_{0}\right)$. It follows from Proposition 2.4 that $2 c V_{-}^{2} \leqq 2 T H_{0}+\ln K$. If we choose $c>T$, this says that $V_{-}$satisfies an estimate $V_{-}^{2} \leqq a^{2}\left(H_{0}+b\right)$ with $a<1$. We may assume that $b \geqq 1$, so that $\left(H_{0}+b\right) \leqq\left(H_{0}+b\right)^{2}$. Thus $V_{-}^{2} \leqq a^{2}\left(H_{0}+b\right)^{2}$. This is the required estimate.

This result justifies the use of the Trotter product formula. This formula has been applied in quantum field theory to show that the field automorphisms converge in the relativistic limit where the space cutoff is removed.

\section{References}

1. Davies, E.B.: Properties of the Green's functions of some Schrödinger operators, to appear.

2. Faris, W.: Product formula for semigroups defined by Friedrichs extensions. Pacific J. Math. 22, 47-70 (1967).

3. Faris, W.: Quadratic forms and essential self-adjointness. Helv. Phys. Acta (to appear). 
4. Glimm,J., Jaffe, A.: Singular perturbations of self-adjoint operators. Commun. Pure Appl. Math. 22, 401-414 (1969).

5. Hoegh-Krohn, R.: A general class of quantum fields without cutoff in two space-time dimensions. Commun. math. Phys. 21, 244-255 (1971).

6. Kato, T.: Perturbation theory for linear operators. Berlin-Heidelberg-New York: Springer 1966.

7. Kato, T.: Schrödinger operators with singular potentials, to appear.

8. Nelson, E.: A quartic interaction in two dimensions. In: Goodman, R., Segal,I.E. (Eds.): Mathematical theory of elementary particles. Cambridge: M.I.T. Press 1966.

9. Nelson, E.: Topics in dynamics I: Flows. Princeton, N.J.: Princeton University Press 1969.

10. Rosen, L.: A $\lambda \phi^{2 n}$ field theory without cutoffs. Commun. math. Phys. 16, 157-183 (1970).

11. Schaefer,H.H.: Topological vector spaces. New York: Macmillan 1966.

12. Segal,I.: Construction of nonlinear local quantum processes: I. Ann. Math. 92, $462-481(1970)$.

13. Segal, I.: Construction of nonlinear local quantum processes: II. Inventiones math. 14, $211-241(1971)$.

14. Simon, B.: Essential self-adjointness of Schrödinger operators with positive potentials. Math. Ann., to appear.

15. Simon, B., Hoegh-Krohn, R.: Hypercontractive semigroups and two dimensional self-coupled Bose fields. J. Funct. Anal. 9, 121-180 (1972).

William G. Faris

Battelle

Advanced Studies Center

Geneva, Switzerland 